\title{
Organ donation and transplantation: a special issue on an ICU gift that lives on
}

\author{
Giuseppe Citerio* ${ }^{*}$
}

๑ 2019 Springer-Verlag GmbH Germany, part of Springer Nature

This thematic issue of Intensive Care Medicine focuses on organ donation and transplantation.

Intensivists play a key role in the identification of the potential donor, in her/his maintenance, in caring for their families, and, after transplantation, in treating the acute postoperative phase.

During the past 50 years, following the definition of a new medical condition, i.e. death by neurological criteria, the medical advances of improved intensive care management and surgical techniques have made organ transplantation a consolidated, cost-effective strategy for end-stage diseases.

In the last decade, most countries have increased their number of deceased organ donors. However, waiting lists continue to exist. To solve this gap, intensivists are integral in identifying possible donors inside and outside of the intensive care unit, optimizing their maintenance and in implementing, when not active, processes of donation after death determined by circulatory criteria. In addition, the utilization of organs from expanded-criteria donors and ex vivo perfusion will increase the availability of organs to be transplanted. All of these aspects are discussed in the dedicated papers of this issue.

We will start with a critical synopsis and key questions in brain death determination more than 50 years after the Harvard Criteria.

After the definition of death, we describe the management of the heart beating donor in the ICU along with a focus on donation after controlled and uncontrolled cardiac death. This latter strategy permitted the expansion of the donor pool in some countries, such as the UK and Spain.

A new, particular challenge in the ICU is the determination of brain death in patients on extracorporeal support, and this has been addressed by a short piece. The

*Correspondence: giuseppe.citerio@unimib.it

School of Medicine and Surgery, University of Milan-Bicocca, Milan, Italy

\section{Springer}

family is not only a bystander during the process, and their involvement in organ donation is crucial. The family is, in fact, at the center of the decision-making process within a limited time frame.

Strategies for expanding the donor pool, including an article on ex vivo perfusion techniques, will be discussed.

After donation, in a series of papers "ten tips for", the authors will present suggestions for the management of the transplanted heart, lung, liver, and kidney patients, along with the management of hematopoietic transplanted patients. Neurologic complications of solid organ transplantation and initial immunosuppression will be discussed in two focus articles. Special circumstances such as organ donation in children, immigrants as donors and transplant recipients, and the challenges of transplantation in people living with HIV will focus on debatable aspects of the process. The final goal of the entire process is to attain good outcomes after organ transplantation and, particularly, an improved quality of life for the patient. We have also inserted the testimony of a transplanted patient who is the focal point of our efforts.

The entire ICM editorial team is confident that this special issue on organ donation and transplantation will clarify some aspects of the process and that it will a helpful resource for the whole ICM community.

Compliance with ethical standards

Conflicts of interest

The authors declare that they have no conflict of Interest.

Ethical approval

An ethical approval was not needed.

\section{Publisher's Note}

Springer Nature remains neutral with regard to jurisdictional claims in published maps and institutional affiliations.

Received: 18 February 2019 Accepted: 19 February 2019

Published online: 26 February 2019 